\title{
Atomic calculations and search for variation of the fine structure constant in quasar absorption spectra
}

\author{
V. A. Dzuba and V. V. Flambaum \\ School of Physics, University of New South Wales, Sydney 2052, Australia
}

(Dated: October 29, 2018)

\begin{abstract}
A brief review of the search for variation of the fine structure constant in quasar absorption spectra is presented. Special consideration is given to the role of atomic calculations in the analysis of the observed data. A range of methods which allow to perform calculations for atoms or ions with different electron structure and which cover practically all periodic table of elements is discussed. Critical compilation of the results of the calculations as well as a review of the most recent results of the analysis are presented.
\end{abstract}

PACS numbers: $31.25 . \mathrm{Eb}, 31.25 . \mathrm{Jf}$

\section{INTRODUCTION}

Theories unifying gravity with other interactions as well as many cosmological models allow for space-time variation of fundamental constants. Experimental search for the manifestation of this variation spans the whole lifetime of the Universe from Big Bang nucleosynthesis to the present-day very precise atomic clock experiments (see, e.g. reviews [1, 2]). An evidence that the fine-structure constant $\alpha\left(\alpha=e^{2} / \hbar c\right)$ might be smaller in early universe has been found in the analysis of quasar absorption spectra [3, 4, 5, 6, 7, 8, 9]. The analyzed data which came from the Keck telescope in Hawaii included three independent samples containing 143 absorption systems which spread over red shift range $0.2<z<4.2$. The fit of the data gives $\delta \alpha / \alpha=$ $(-0.543 \pm 0.116) \times 10^{-5}[10]$. If one assumes the linear dependence of $\alpha$ on time, the fit of the data gives $d \ln \alpha / d t=(6.40 \pm 1.35) \times 10^{-16}$ per year (over time interval about 12 billion years).

A very extensive search for possible systematic errors 11] has shown that known systematic effects can not explain the result. Although it is still not completely excluded that the effect may be imitated by a large change of abundances of isotopes in last 10 billion years, it would need a very unlikely "conspiracy" between several elements. It had been checked that different isotopic abundances for any single element can not imitate the observed effect.

Recently two other groups of researchers [12, 13, 14] applied the same method of the analysis to a different set of data obtained from the VLT telescope in Chile and reported no variation of $\alpha$. There was an intensive debate in the literature about possible reasons for disagreement. It was argued in particular that at least part of the disagreement may be attributed to the spatial variation of $\alpha$. This argument is based on the fact that Keck telescope is in the Northern hemisphere while VLT telescope is in the Southern hemisphere.

The results of [12] were recently questioned in Refs. 15, 16]. Re-analysis of the data of Ref. [12] revealed flawed parameter estimation methods. The authors of [15, 16] claim that the same spectral data fitted more accurately give $\delta \alpha / \alpha=(-0.64 \pm 0.36) \times 10^{-5}$ rather than $\delta \alpha / \alpha=$ $(-0.06 \pm 0.06) \times 10^{-5}$ as in Ref. [12]. However, even this new result may require further revision.

All the results discussed above were obtained with the use of the so called many-multiplet (MM) method which was proposed in Ref. [17]. This method uses atomic calculations $18,19,20,21,22,23,24,25,26,27$ to relate the change in the value of the fine structure constant to the change in the frequencies of atomic transitions. It is more than an order of magnitude more sensitive to the variation of the fine structure constant than the analysis of the fine structure intervals used for this purpose before.

In present paper we review the methods of atomic calculations used in the search for the variation of the fine structure constant and present critical compilation of the most accurate results of the calculations and discuss some future directions.

\section{ATOMIC CALCULATIONS}

In atomic units $\alpha=1 / c$, were $c$ is speed of light, and $\alpha=0$ corresponds to non-relativistic limit. Therefore, to reveal the dependence of atomic frequencies on $\alpha$ we need to perform relativistic calculations based on the Dirac equation. Doing this way we include leading relativistic corrections of the order $(Z \alpha)^{2}$. The role of smaller corrections, such as Breit and quantum electrodynamic (QED) corrections will be discussed in section IIE

It is convenient to present the dependence of atomic frequencies on the fine-structure constant $\alpha$ in the vicinity of its physical value $\alpha_{0}$ in the form

$$
\omega(x)=\omega_{0}+q x,
$$

where $\omega_{0}$ is the present laboratory value of the frequency and $x=\left(\alpha / \alpha_{0}\right)^{2}-1, q$ is the coefficient which is to be found from atomic calculations. Note that

$$
q=\left.\frac{d \omega}{d x}\right|_{x=0} .
$$


TABLE I: $A b$ initio methods of atomic calculations depending on the number of valence electrons $\left(N_{v}\right)$.

\begin{tabular}{lll}
\hline$N_{v}$ & Method & Accuracy \\
\hline 1 & MBPT + all-order sums & $0.1-1 \%$ \\
$2-8$ & MBPT + Configuration interaction (CI+MBPT) & $1-10 \%$ \\
$2-15$ & Configuration interaction (CI) & $10-20 \%$ \\
\hline
\end{tabular}

To calculate this derivative numerically we use

$$
q \approx \frac{\omega(+\delta)-\omega(-\delta)}{2 \delta} .
$$

and vary the value of $\alpha$ in the computer code.

We use relativistic Hartree-Fock method as a starting point of all calculations. The radial equation for singleelectron orbitals has the form (atomic units)

$$
\begin{gathered}
\frac{d f_{v}}{d r}+\frac{\kappa_{v}}{r} f_{v}(r)-\left[2+\alpha^{2}\left(\epsilon_{v}-\hat{V}_{H F}\right)\right] g_{v}(r)=0 \\
\frac{d g_{v}}{d r}-\frac{\kappa_{v}}{r} f_{v}(r)+\left(\epsilon_{v}-\hat{V}_{H F}\right) f_{v}(r)=0
\end{gathered}
$$

here $\kappa=(-1)^{l+j+1 / 2}(j+1 / 2)$, index $v$ replaces the threenumber set of the principal quantum number, and total and angular momentum: $n, j, l . \hat{V}_{H F}$ is the Hartree-Fock potential.

Equations (4) with $\alpha=\alpha_{0} \sqrt{\delta+1}$ are solved selfconsistently for all core states to find Hartree-Fock potential of the atomic core. Then this potential is used to calculate a full set of single-electron orbitals for the states above the core.

After single-electron states are calculated, the actual choice of the methods to calculate many-electron states of valence electrons depends on the number of valence electrons. Table I summarizes the methods used in the calculations. These methods will be discussed in more detail in following sections.

\section{A. Atoms with one external electron}

Atoms and ions of astrophysical interest which can be considered as systems with one external electron above closed shells in both ground and excited states include C IV, O VI, Na I, Mg II, Al III, Si IV, Ca II, Zn II and Ge II. All calculations for these atoms and ions were performed in the $V^{N-1}$ approximation in which Eq. (4) are solved for the closed-shell core with the external electron removed. The states of valence electron are calculated in the $V^{N-1}$ potential of the frozen core. Correlations are also included using different numerical techniques. In most of the calculations (see, e.g. Ref. 18]) the correlation potential method [28] was used.

In this approach the correlation potential $\hat{\Sigma}$, which describes correlations between external and core electrons, is calculated in the lowest, second-order of the manybody perturbation theory (MBPT) using the B-spline basis set [29]. All four second-order diagrams for $\hat{\Sigma}$ are presented on Fig. 1.
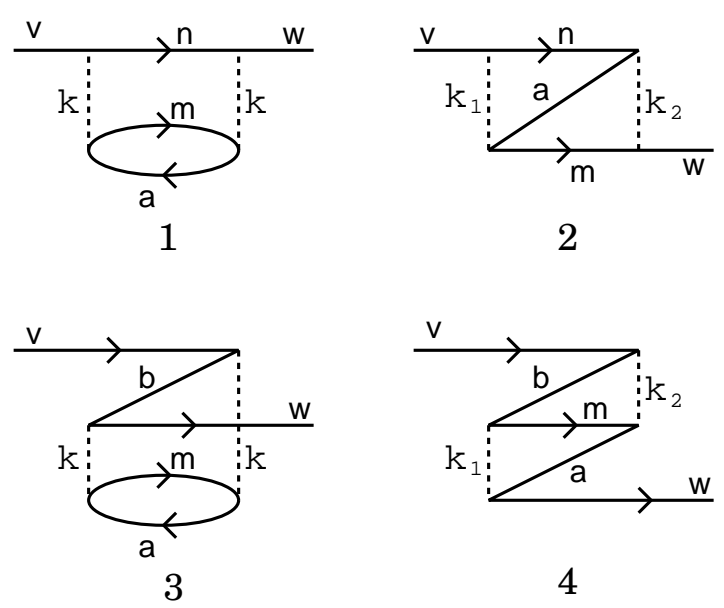

FIG. 1: Second-order diagrams for the matrix elements $\left\langle v\left|\hat{\Sigma}_{1}^{(2)}\right| w\right\rangle$ of the single-electron correlation operator $\hat{\Sigma}_{1}^{(2)}$.

The $\hat{\Sigma}$ operator is a non-local operator similar to the Hartree-Fock exchange potential. It is used in the equations for the states of an external electron to calculate the so-called Brueckner orbitals (BO). The orbitals and corresponding energies include all second-order correlations as well as higher-order correlations which correspond to $\langle\hat{\Sigma}\rangle^{2},\langle\hat{\Sigma}\rangle^{3}$, etc. The accuracy for the energies in this approach is usually few per cent or better.

Correlation potential method with the second-order $\hat{\Sigma}$ does not include dominating higher-order correlations. The effect of the higher-order correlations on the $q$ factors for mono-valent atoms of astrophysical interest were studied in detail in Ref.[25] within the single-double coupled cluster approach and were found to be small.

\section{B. Atoms with open $s$ and $p$ shells}

Atoms or ions with two or more electrons on open $s$ and/or $p$ shells are next in the complexity of the calculations. Systems found in astrophysical observations include C I, C II, C III, O I, O II, O III, $\mathrm{O}$ IV, Mg I and Al II. We perform calculations for such systems with the use of the configuration interaction method combined with the many-body perturbation theory (CI+MBPT) [30]. Configuration interaction (CI) technique is used for accurate treatment of the interaction between valence electrons while the MBPT is used to include correlations between core and valence electrons. Similar to the case of atoms with one external electron, correlation operator $\hat{\Sigma}$ is used to describe the core-valence correlations. However, it now consists of at least two parts. $\hat{\Sigma}_{1}$ describes correlations between an external electron and the electrons in the core, while $\hat{\Sigma}_{2}$ describes correlation correction (screening) to the Coulomb interaction between two external electrons caused by the core electrons. The $\hat{\Sigma}_{1}$ operator is similar to the $\hat{\Sigma}$ operator used for atoms with one external 
electron. The actual form for of the $\hat{\Sigma}$ operator depends on the choice of the potential in which core states are calculated. The simplest form is in the so-called $V^{N-M}$ approximation [31, 32] which is a generalization of the $V^{N-1}$ approximation used for atoms with one external electron.

In this approximation the $M$ external electrons are excluded from the initial Hartree-Fock procedure for the core to make sure that the effective potential of the CI Hamiltonian for the valence states and the potential in which core electrons are calculated are the same. This is the key for the simplest form of the MBPT. This approach gives good results for atoms with open $s$ or $p$ shells because electrons of these shells are localized on large distances and have very small effect on the wave functions of the core states (in spine of large effect on their energies, see Refs. 31, 32 for details).

In the $V^{N-M}$ approximation the $\hat{\Sigma}_{1}$ operator is identical to the $\hat{\Sigma}$ operator used for atoms with one external electron. Corresponding diagrams are presented on Fig. 1. Diagrams for $\hat{\Sigma}_{2}$ are presented on Fig. 2 .

If the potential in which core states are calculated is different from the $V^{N-M}$ potential, then both $\hat{\Sigma}_{1}$ and $\hat{\Sigma}_{2}$ include the so-called subtraction diagrams which account for this difference (see Ref. 30] for details).

The effective CI Hamiltonian for the valence electrons has the form

$$
\hat{H}^{C I}=\sum_{i=1}^{M}\left[\hat{h}_{0 i}+\hat{\Sigma}_{1 i}\right]+\sum_{i<j}^{M}\left[\frac{1}{r_{i j}}+\hat{\Sigma}_{2 i j}\right],
$$

where $\hat{h}_{0}$ is a single-electron Hartree-Fock Hamiltonian which corresponds to Eq. (4).

The CI equations

$$
\hat{H}^{C I} \Psi\left(r_{1}, \ldots, r_{M}\right)=E \Psi\left(r_{1}, \ldots, r_{M}\right)
$$

are solved by matrix diagonalization using the the manyelectron basis states constructed from the B-splines. Therefore, the B-spline basis set serves the dual purpose in the calculations: to calculate the correlation operator $\hat{\Sigma}$ and to solve the CI equations.

The details of the calculations vary slightly from atom to atom. For example, $\hat{\Sigma}$ was not included for light atoms like carbon. Here relativistic corrections are small and high accuracy of the calculations is not needed.

\section{Atoms with open $d$ or $f$ shells}

Atoms with open $d$ and $f$ shells are most difficult for calculations. This is mostly due to large number of electrons which enter the CI calculations. This makes it practically impossible to saturate the basis with an arbitrary choice of single-electron basis states.

Also, accurate treatment of the core-valence correlations is much more difficult. First, the $V^{N-M}$ approximation is not valid because $d$ and $f$ electrons are inside

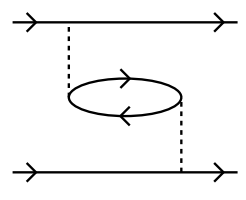

1

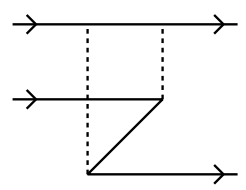

4

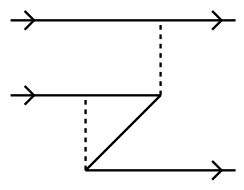

2

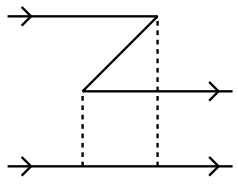

5

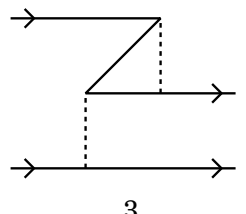

3

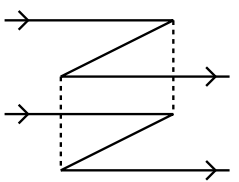

6
FIG. 2: Second-order diagrams for double-electron correlation operator $\hat{\Sigma}_{2}^{(2)}$.

the core and cannot be neglected in the initial HF calculations for the core. Therefore, subtraction diagrams must be included. Second, for atoms with more than two valence electrons, a new kind of $\hat{\Sigma}$ operator appear even in the lowest second-order of the MBPT, the threeelectron operator $\hat{\Sigma}_{3}$ (see Ref. [30] for details). The most detailed study of an atom with open $d$-shell, which used large basis, all three $\hat{\Sigma}$-operators $\hat{\Sigma}_{1}, \hat{\Sigma}_{2}$ and $\hat{\Sigma}_{3}$ and Breit interaction were performed in Ref. [33] for the Fe II ion.

We use a less sophisticated approach which still gives reasonably accurate results [18, 19, 20, 21, 27]. It is based on the CI technique with simplified treatment of the corevalence correlations (see below) and different choice of the basis in different calculations for the valent states. In our early calculations [18, 19, 20, 21] we used either a Bspline basis set or a set of orbitals which was constructed using a recurrent procedure suggested in Ref. [34]. In this procedure an virtual orbital is constructed from a lower one by multiplying it by distance $r$ and orthogonalizing it to all lower orbitals (see also Ref. 30]).

In the most recent work [27] we used a more sophisticated approach in which a set of non-orthogonal HartreeFock valence states was constructed. The self-consistent $\mathrm{HF}$ procedure was done for each configuration of interest separately. Only one single-electron basis function in each partial wave is included in the CI for each configuration. However, this functions represent good initial approximations since they come from the self-consistent HF calculations.

To include core-valence correlations $\hat{\Sigma}_{1}$ is replaced by a correction to the local part of the HF potential of the atomic core

$$
\delta V=-\frac{\alpha_{p}}{2\left(r^{4}+a^{4}\right)}
$$

Here $\alpha_{p}$ is polarization of the core and $a$ is a cut-off parameter (we use $a=a_{B}$ ). The form of the $\delta V$ is chosen to coincide with the standard polarization potential on large distances $\left(-\alpha_{p} / 2 r^{4}\right)$. The $\alpha_{p}$ is treated as a fitting parameter and values of $\alpha_{p}$ for each configuration are chosen to reproduce their position in experimental spectrum. 
The $\hat{\Sigma}_{2}$ and $\hat{\Sigma}_{3}$ operators are either neglected or simulated by introducing screening parameters for Coulomb integrals. It is usually assumed that the screening parameters $f_{k}$ depend only on the multipolarity $k$ of the Coulomb interaction and their values are chosen to have better agreement with the experimental energies. It is also assumed that all fitting parameters $\alpha_{p}$ and $f_{k}$ do not depend on $\alpha$. This is justified because the fitting represents only small correction to the energies.

The effective CI Hamiltonian has the form

$$
\hat{H}^{C I}=\sum_{i=1}^{M}\left[\hat{h}_{0 i}+\delta V_{i}\right]+\sum_{i<j}^{M} \frac{1}{r_{i j}} .
$$

It can be obtained from (5) by dropping $\hat{\Sigma}_{2}$ and replacing $\hat{\Sigma}_{1}$ by $\delta V$ [see Eq. (7)].

\section{Level pseudo-crossing}

Calculations may be complicated significantly by the phenomenon of level pseudo-crossing [19, 20]. In atoms with dense spectrum the states of the same total momentum $J$ and parity and separated by small energy interval may be strongly mixed which in turn may lead to instability of the calculations of the $q$ coefficients. This may be considered as level pseudo-crossing in the vicinity of the physical value of $\alpha$ if energies of these two states are considered as functions of $\alpha^{2}$. In two-level approximation the $q$ coefficients for each state are

$$
\begin{aligned}
& q_{1}=\cos ^{2} \phi q_{1}^{(L S)}+\sin ^{2} \phi q_{2}^{(L S)} \\
& q_{2}=\sin ^{2} \phi q_{1}^{(L S)}+\cos ^{2} \phi q_{2}^{(L S)},
\end{aligned}
$$

where $\phi$ is the mixing angle and $q_{1}^{(L S)}$ and $q_{2}^{(L S)}$ are the $q$ coefficients for the states which correspond to the values of $\alpha$ far from the crossing. Very often atomic states in the absence of crossing are well described by the $L S$ coupling scheme which is indicated by the $(L S)$ superscripts in Eq. 9. If $q_{1}^{(L S)}$ and $q_{2}^{(L S)}$ are significantly different than the values of $q_{1}$ and $q_{2}$ strongly depend on the mixing angle $\phi$ which leads to instability of the results of calculations.

The best way of dealing with this instability is to use experimental values of the Landé $g$-factors. In the two level approximation the $g$-factors of two states are given by the formula very similar to Eq. (9):

$$
\begin{aligned}
& g_{1}=\cos ^{2} \phi g_{1}^{(L S)}+\sin ^{2} \phi g_{2}^{(L S)}, \\
& g_{2}=\sin ^{2} \phi g_{1}^{(L S)}+\cos ^{2} \phi g_{2}^{(L S)},
\end{aligned}
$$

with the same mixing angle $\phi$ as in (9) and with $g_{1}^{(L S)}$ and $g_{2}^{(L S)}$ given by

$$
g^{(L S)}=1+\frac{J(J+1)-L(L+1)-S(S+1)}{2 J(J+1)},
$$

where $J, L, S$ are total and angular momentums and spin of the states. Eqs. (10[11) can be used to find the mixing angle $\phi$ which than is used to correct the calculated values of $q_{1}$ and $q_{2}$.

Note that it follows from (9) that the sum $g_{1}+g_{2}=$ $g_{1}^{(L S)}+g_{2}^{(L S)}$ and does not depend on $\phi$. This can be used to check the accuracy of the two-level approximation.

This scheme cannot be used if experimental values of $g$-factors are not known or if $g_{1}+g_{2} \neq g_{1}^{(L S)}+g_{2}^{(L S)}$ or if $g_{1}^{(L S)}=g_{2}^{(L S)}$. If experimental $g$-factors are known but $g_{1}+g_{2} \neq g_{1}^{(L S)}+g_{2}^{(L S)}$ then a multilevel consideration may help as explained in Ref. [20]. Otherwise the only way to get reliable results is to fit the energies to experimental values to the accuracy sufficiently better than the small energy interval between strongly mixed states (see, e.g. [27]).

\section{E. Breit and QED corrections}

So far we have considered relativistic corrections to atomic energies which come from the Dirac equation. These corrections have an order of $(Z \alpha)^{2}$. There are however other smaller corrections like Breit and quantum electrodynamic corrections. It is important to understand the role these corrections may play in the search for variation of $\alpha$. Breit corrections are proportional to $\alpha^{2}$ but smaller than 2 power of nuclear charge $Z$. Its relative contribution is therefore larger for light atoms. In contrast, the QED corrections are proportional to $\alpha^{3}$ but stronger than $Z^{2}$ functions of $Z$. Their relative contribution is larger for heavy atoms. The role of the Breit interaction was studied in detail in Ref. 26] for mono and double-valent electron atoms and ions of astrophysical interest and in Ref. [33] for Fe II. The role of the QED correction has not been studied yet but expected to be small.

The following form of the Breit operator is used in the relativistic calculations for many-electron atoms (atomic units)

$$
\hat{H}^{B}=-\frac{\hat{\alpha}_{1} \cdot \hat{\alpha}_{2}+\left(\hat{\alpha}_{1} \cdot \hat{\mathbf{n}}\right)\left(\hat{\alpha}_{2} \cdot \hat{\mathbf{n}}\right)}{2 r} .
$$

Here $\mathbf{r}=\hat{\mathbf{n}} r, r$ is the distance between electrons and $\hat{\alpha}_{i}$ is the $\alpha$-matrix of the corresponding electrons. This is a low frequency limit of the relativistic correction to the Coulomb interaction between electrons. It contains magnetic interaction and retardation.

It is important to include $\hat{H}^{B}$ into Hartree-Fock Hamiltonian to take into account the effect of Breit interaction on the self-consistent field (relaxation effect). for atoms with two or more external electrons Breit term is also included as a correction to the Coulomb interaction in the CI Hamiltonian.

It turns out [26, 33] that Breit corrections to the $q$ values are relatively small and unlikely affect the analysis of quasar spectra in terms of variation of $\alpha$. However, it 
TABLE II: Energies of the transitions from the ground state of single-valent atoms and ions of astrophysical interest and corresponding relativistic $q$ coefficients $\left(\mathrm{cm}^{-1}\right)$.

\begin{tabular}{|c|c|c|c|c|c|}
\hline & Atom & State & $\mathrm{E}(\operatorname{expt})$ & $\mathrm{E}($ calc $)$ & $q$ \\
\hline \multirow{2}{*}{\multicolumn{2}{|c|}{$6 \mathrm{C} \mathrm{IV}$}} & $2 p_{1 / 2}$ & 64484 & 64504 & $115(2)$ \\
\hline & & $2 p_{3 / 2}$ & 64591 & 64636 & $222(2)$ \\
\hline \multirow{4}{*}{\multicolumn{2}{|c|}{$11 \mathrm{Na} \mathrm{I}$}} & $3 p_{1 / 2}$ & 16956 & 16961 & $45(0)$ \\
\hline & & $3 p_{3 / 2}$ & 16973 & 16979 & $62(0)$ \\
\hline & & $4 p_{1 / 2}$ & 30267 & 30066 & $57(1)$ \\
\hline & & $4 p_{3 / 2}$ & 30273 & 30072 & $51(1)$ \\
\hline \multirow[t]{4}{*}{12} & Mg II & $3 p_{1 / 2}$ & 35669 & 87 & $121(1)$ \\
\hline & & $3 p_{3 / 2}$ & 357 & 35784 & $212(1)$ \\
\hline & & $4 p_{1 / 2}$ & 80620 & 80463 & 161(1) \\
\hline & & $4 p_{3 / 2}$ & & 96 & 192(1) \\
\hline \multirow[t]{4}{*}{13} & $\mathrm{Al}$ III & $3 p_{1 / 2}$ & 536 & 23 & $224(1)$ \\
\hline & & $3 p_{3 / 2}$ & & & $458(2)$ \\
\hline & & $4 p_{1 / 2}$ & 143633 & 143 & $337(2)$ \\
\hline & & $4 p_{3 / 2}$ & 143714 & 143623 & $417(3)$ \\
\hline \multirow{4}{*}{\multicolumn{2}{|c|}{14 Si IV }} & $3 p_{1 / 2}$ & 712 & 71352 & $361(2)$ \\
\hline & & $3 p_{3 / 2}$ & 717 & 36 & $823(2)$ \\
\hline & & $4 p_{1 / 2}$ & 218267 & 218226 & $597(4)$ \\
\hline & & & 218429 & 218397 & $760(4)$ \\
\hline \multirow{2}{*}{\multicolumn{2}{|c|}{$20 \mathrm{Ca}$ II }} & $4 p_{1 / 2}$ & 25192 & 25086 & $222(1)$ \\
\hline & & $4 p_{3 / 2}$ & 254 & 25315 & $446(3)$ \\
\hline \multirow{2}{*}{\multicolumn{2}{|c|}{$30 \mathrm{Zn} \mathrm{II}$}} & $4 p_{1 / 2}$ & 48481 & 48721 & $1541(7)$ \\
\hline & & $4 p_{3 / 2}$ & 49355 & 49606 & $2452(13)$ \\
\hline
\end{tabular}

is useful to include them for more accurate results. For example, as it was demonstrated in Ref. [26] inclusion of Breit interaction significantly improves the agreement between theoretical and experimental fine structure making the result to be more accurate and therefore more reliable.

Similar role is expected for the QED corrections. But it is the subject of future work. An adequate method to include leading QED corrections to the energies of manyelectron atoms has been developed in Ref. 35].

\section{RESULTS OF CALCULATIONS}

The results of the calculated $q$-values for lines which were observed in quasar absorption spectra are presented in Tables II, III and IV. Calculated and experimental energies are also presented to illustrate the accuracy of calculations. Experimental energies are taken from the NIST website [36]. Estimations of uncertainties for the values of $q$-factors are based on the sensitivity of the results to variations of the calculation scheme and on the comparison of the experimental and theoretical energies and fine structure intervals. In cases when several calculations are available only latest most accurate results are presented. For the case of Fe II we also present the results of independent calculations by the St. Petersburg group 33 .

The analysis of the values of the $q$-factors reveal an interesting picture. These values vary strongly from atom to atom and from one transition to another. The values can be very small or can be large positive or large negative. This is due to the effect of several factors which can be qualitatively illustrated by the value of the relativistic energy shift of a single-electron state [18]

$$
\Delta_{n}=\frac{E_{n}}{\nu}(Z \alpha)^{2}\left[\frac{1}{j+1}-C(Z, j, l)\right],
$$

where $E_{n}$ is the energy of the single-electron state $n, \nu$ is the effective principal quantum number $\left(\nu=1 / \sqrt{-2 E_{n}}\right)$, $j$ is the total momentum of the state and constant $C$ $(C \approx 0.6)$ simulates the effect of exchange interaction and other many-body effects. The value of the $q$-factor for a particular atomic transition can be roughly estimated assuming that the transition involves the change of a state of just one electron. Then

$$
q \approx \Delta_{n}-\Delta_{n^{\prime}}
$$

where state $n^{\prime}$ is above state $n$.

The first and most obvious feature of the $q$-factors is its $Z^{2}$ dependence on the nuclear charge $Z$. However, it also depends, e. g. on the ionization degree. This is because the absolute value of the single electron energy $E_{n}$ (13) is larger for ions. Also, dependence of the energy shift (13) on the total electron momentum $j$ suggests that the value of $q$ is likely to by positive for the $s-p$ transitions and negative for the $p-d$ transitions.

All these features of the $q$-factor values are reproduced in the accurate numerical calculations (see Tables IIIII and IV]. This complex dependence of atomic frequencies on $\alpha$ is very important for study of possible systematics. It is very unlikely that any other unknown effect exhibits exactly the same features. Therefore, if the results of the analysis based on different transitions give the same result for variation of $\alpha$ then the mimic of the effect by any systematic is very improbable.

Theoretic uncertainty for the values of $q$ varies significantly from atom to atom. The most accurate results are for atoms which can be considered as atoms with single valence electron above closed shells in both ground and excited states. The results for such atoms are presented in Table [II] They were obtained using second-order MBPT [26] as well as the single-double coupled cluster approximation combined with the thirdorder MBPT [25]. Small uncertainty is due to excellent agreement between different approaches and between experimental and theoretical data for the energies and fine structure intervals. Breit interaction was also included in Ref. [26]. Although Breit contributions are very small for the purposes of the analysis they are larger than the uncertainty of the calculation of the correlations. It was demonstrated in Ref. [26] that inclusion of Breit contributions improve significantly theoretical fine structure brining it to almost perfect agreement with the experiment.

Table III presents results for Fe I and Fe II. Iron is one of the most important elements used in the analysis. It is used more often than most of other elements and it 
TABLE III: Energies of the transitions from the ground state of Fe I and Fe II and corresponding relativistic $q$ coefficients $\left(\mathrm{cm}^{-1}\right)$.

\begin{tabular}{|c|c|c|c|c|c|c|c|c|c|c|}
\hline \multirow{2}{*}{$\begin{array}{l}\text { Atom } \\
\text { or ion }\end{array}$} & \multicolumn{3}{|c|}{ Ground state } & \multicolumn{3}{|c|}{ Upper state } & \multicolumn{2}{|c|}{ Energy } & \multicolumn{2}{|r|}{$q$} \\
\hline & Config. & Term & $J$ & Config. & Term & $J$ & Expt.[36] & Calc. & [27] & [33] \\
\hline \multirow[t]{8}{*}{ Fe I } & $3 d^{6} 4 s^{2}$ & ${ }^{5} \mathrm{D}$ & 4 & $3 d^{6} 4 s 4 p$ & ${ }^{5} \mathrm{D}^{O}$ & 4 & 25899 & 26428 & $999(300)$ & \\
\hline & & & & $3 d^{6} 4 s 4 p$ & ${ }^{5} \mathrm{~F}^{o}$ & 5 & 26874 & 27432 & $880(260)$ & \\
\hline & & & & $3 d^{6} 4 s 4 p$ & ${ }^{5} \mathrm{P}^{o}$ & 3 & 29056 & 29340 & $859(260)$ & \\
\hline & & & & $3 d^{5} 4 s^{2} 4 p$ & ${ }^{5} \mathrm{D}^{o}$ & 4 & 33095 & 32680 & $2494(750)$ & \\
\hline & & & & $3 d^{5} 4 s^{2} 4 p$ & ${ }^{5} \mathrm{D}^{o}$ & 3 & 33507 & 33134 & $3019(900)$ & \\
\hline & & & & $3 d^{5} 4 s^{2} 4 p$ & ${ }^{5} \mathrm{~F}^{o}$ & 5 & 33695 & 32522 & $2672(800)$ & \\
\hline & & & & $3 d^{5} 4 s^{2} 4 p$ & ${ }^{5} \mathrm{D}^{o}$ & 4 & 39625 & 39544 & $1680(500)$ & \\
\hline & & & & $3 d^{5} 4 s^{2} 4 p$ & ${ }^{5} \mathrm{~F}^{o}$ & 5 & 40257 & 40194 & $1042(300)$ & \\
\hline \multirow[t]{7}{*}{$\mathrm{Fe} \mathrm{II}$} & $3 d^{6} 4 s$ & ${ }^{6} \mathrm{D}$ & $9 / 2$ & $3 d^{6} 4 p$ & ${ }^{6} \mathrm{D}^{o}$ & $9 / 2$ & 38458 & 38352 & $1330(150)$ & $1410(60)$ \\
\hline & & & & $3 d^{6} 4 p$ & ${ }^{6} \mathrm{D}^{o}$ & $7 / 2$ & 38660 & 38554 & $1490(150)$ & $1540(40)$ \\
\hline & & & & $3 d^{6} 4 p$ & ${ }^{6} \mathrm{~F}^{o}$ & $11 / 2$ & 41968 & 41864 & $1460(150)$ & $1550(60)$ \\
\hline & & & & $3 d^{6} 4 p$ & ${ }^{6} \mathrm{~F}^{o}$ & $9 / 2$ & 42114 & 42012 & $1590(150)$ & $1660(60)$ \\
\hline & & & & $3 d^{6} 4 p$ & ${ }^{6} \mathrm{P}^{o}$ & $7 / 2$ & 42658 & 42715 & $1210(150)$ & $1540(400)$ \\
\hline & & & & $3 d^{6} 4 p$ & ${ }^{4} \mathrm{~F}^{o}$ & $7 / 2$ & 62065 & 65528 & $1100(300)$ & $1560(500)$ \\
\hline & & & & $3 d^{5} 4 s 4 p$ & ${ }^{6} \mathrm{P}^{o}$ & $7 / 2$ & 62171 & 65750 & $-1300(300)$ & $-1030(300)$ \\
\hline
\end{tabular}

is also most studied theoretically. First analysis of the quasar absorption spectra compared shift of frequencies of Fe II to those of Mg I and Mg II. The values of the $q$ coefficients for magnesium are small compared to those for Fe II. Therefore, one can say they were used as "anchors" against which the shift of Fe II lines was measured. When it was realized that Fe II has also large negative shifters (see last line of Table III) it was suggested that Fe II alone can be used in the analysis by comparing lines with positive and negative values of $q$. This may help to eliminate certain types of systematic errors [33].

Calculations for Fe II were carried out by means of the CI method in Refs. [18, 20]. The more accurate results from the later work are included in the Table. The Table also presents the results of independent calculations by the St. Petersburg group 33]. This is the most detailed and accurate calculations which include core-valence correlations and Breit corrections. The results of both calculations agree within the declared accuracy. Accuracy is high for lower states but deteriorates significantly higher in the spectrum due to increasing configuration mixing and difficulty in the achieving of basis saturation for as many as seven external electrons.

Lines of neutral iron has not been used in the analysis yet. However, the lines presented in Table III are observed in the quasar absorption spectra and are planed to be included into analysis. Therefore we have recently calculated the $q$ coefficients for them [27]. It turned out that the values of $q$ are large and for some lines they are even larger than for Fe II. This is due to strong configuration mixing which makes the transitions to be effectively two-electron transitions (see Ref. [27] for details). This makes Fe I a good candidate for the inclusion into the analysis.

The results for other atoms are presented in Table IV. The calculations were carried out in works of Refs. 18, 19, 20, 21, 22, 24]. See these works for more detailed information. Here we present only the most commonly used lines and the latest more accurate calculations.

\section{FUTURE DIRECTIONS}

The analysis of quasar absorption spectra has already produced very important results putting strong constrains on possible space-time variation of the fine structure constants $\alpha$ and hinting that $\alpha$ might be smaller in early epoch. However the results are controversial and more work is needed to prove or dismiss the contradicting claims. Resolving the disagreement between the analysis of the Keck and VLT data seems to be the most important thing at the moment. This should involve careful cross re-analysis of the data by independent groups of experts.

It is also important to include more data into the analysis to improve statistics significantly. For example, having sufficient statistics for different lines with different shifts would allow to compare the results which come from the analysis of each line separately and therefore exclude many possible systematics. Also, improving statistics may allow to study variation of the fine structure constant as a function of the red-shift parameter $z$ or as a function of the position in the sky (space variation).

There are probably many more lines observed in quasar absorption spectra but still not used in the analysis. Inclusion of this data may also add important information about $\alpha$ variation. For example, calculations for lines of Fe I which are available for the analysis reveal that the frequencies shifts for these lines due to change of $\alpha$ are large and significantly different for different lines. Therefore, if corresponding frequency shifts are observed it would be hard to attribute them to anything but variation of $\alpha$.

Another example is the inclusion of the weak M1 or E2 
TABLE IV: Energies of the transitions from the ground state of atoms and ions of astrophysical interest and corresponding relativistic $q$ coefficients $\left(\mathrm{cm}^{-1}\right)$.

\begin{tabular}{|c|c|c|c|c|c|c|c|c|}
\hline \multirow{2}{*}{\multicolumn{2}{|c|}{$\begin{array}{l}Z \begin{array}{l}\text { Atom } \\
\text { or ion }\end{array}\end{array}$}} & \multirow{2}{*}{\multicolumn{2}{|c|}{$\begin{array}{c}\text { Ground } \\
\text { state }\end{array}$}} & \multirow{2}{*}{\multicolumn{2}{|c|}{$\begin{array}{l}\text { Upper } \\
\text { state }\end{array}$}} & \multicolumn{2}{|c|}{ Energy } & \multirow[t]{2}{*}{$q$} \\
\hline & & & & & & Expt.[36] & Calc. & \\
\hline \multirow{5}{*}{\multicolumn{2}{|c|}{$6 \mathrm{C} \mathrm{I}$}} & $2 s^{2} 2 p^{2}$ & ${ }^{3} \mathrm{P}_{0}$ & $2 s 2 p^{3}$ & ${ }^{3} \mathrm{D}_{3}^{o}$ & 64087 & 66722 & $151(60)$ \\
\hline & & & & $2 s 2 p^{3}$ & ${ }^{3} \mathrm{D}_{1}^{o}$ & 64090 & 66712 & $141(60)$ \\
\hline & & & & $2 s 2 p^{3}$ & ${ }^{3} \mathrm{D}_{2}^{o}$ & 64091 & 66716 & $145(60)$ \\
\hline & & & & $2 s 2 p^{3}$ & ${ }^{3} \mathrm{P}_{1}^{o}$ & 75254 & 75978 & $111(60)$ \\
\hline & & & & $2 s 2 p^{3}$ & ${ }^{3} \mathrm{~S}_{1}^{o}$ & 105799 & 100170 & $130(60)$ \\
\hline \multirow{4}{*}{\multicolumn{2}{|c|}{$6 \mathrm{C} \mathrm{II}$}} & $2 s^{2} 2 p$ & ${ }^{2} \mathrm{P}_{1 / 2}^{o}$ & $2 s^{2} 2 p$ & ${ }^{2} \mathrm{P}_{3 / 2}^{o}$ & 63 & 74 & $63(1)$ \\
\hline & & & & $2 s 2 p^{2}$ & ${ }^{2} \mathrm{D}_{5 / 2}$ & 74930 & 76506 & $179(20)$ \\
\hline & & & & $2 s 2 p^{2}$ & ${ }^{2} \mathrm{D}_{3 / 2}$ & 74933 & 76503 & $176(20)$ \\
\hline & & & & $2 s 2 p^{2}$ & ${ }^{2} \mathrm{~S}_{1 / 2}$ & 96494 & 97993 & $161(30)$ \\
\hline 6 & 6 C III & $2 s^{2}$ & ${ }^{1} \mathrm{~S}_{0}$ & $2 s 2 p$ & ${ }^{1} \mathrm{P}_{1}^{o}$ & 102352 & 103955 & 163(1) \\
\hline 7 & $7 \mathrm{~N} \mathrm{~V}$ & $2 s$ & ${ }^{2} \mathrm{~S}_{1 / 2}$ & $2 p$ & ${ }^{2} \mathrm{P}_{3 / 2}^{o}$ & 80722 & 81607 & $492(50)$ \\
\hline \multirow{3}{*}{\multicolumn{2}{|c|}{8 O II }} & $2 s^{2} 2 p^{3}$ & ${ }^{4} \mathrm{~S}_{3 / 2}^{o}$ & $2 s 2 p^{4}$ & ${ }^{4} \mathrm{P}_{5 / 2}$ & 119873 & 122620 & $346(50)$ \\
\hline & & & & $2 s 2 p^{4}$ & ${ }^{4} \mathrm{P}_{3 / 2}$ & 120000 & 122763 & $489(50)$ \\
\hline & & & & $2 s 2 p^{4}$ & ${ }^{4} \mathrm{P}_{1 / 2}$ & 120083 & 122848 & $574(50)$ \\
\hline \multirow{2}{*}{\multicolumn{2}{|c|}{$8 \mathrm{O} \mathrm{III}$}} & $2 s^{2} 2 p^{2}$ & ${ }^{3} \mathrm{P}_{0}$ & $2 s 2 p^{3}$ & ${ }^{3} \mathrm{D}_{1}^{o}$ & 120058 & 121299 & $723(50)$ \\
\hline & & & & $2 s 2 p^{3}$ & ${ }^{3} \mathrm{P}_{2}^{o}$ & 142382 & 143483 & $726(50)$ \\
\hline 8 & $8 \mathrm{O} \mathrm{IV}$ & $2 s^{2} 2 p$ & ${ }^{2} \mathrm{P}_{1 / 2}^{o}$ & $2 s 2 p^{2}$ & ${ }^{2} \mathrm{D}_{3 / 2}$ & 126950 & 129206 & $840(50)$ \\
\hline \multirow{2}{*}{\multicolumn{2}{|c|}{$8 \mathrm{O} \mathrm{VI}$}} & $2 s$ & ${ }^{2} \mathrm{~S}_{1 / 2}$ & $2 p$ & ${ }^{2} \mathrm{P}_{1 / 2}^{o}$ & 96375 & 96501 & $309(50)$ \\
\hline & & & & $2 p$ & ${ }^{2} \mathrm{P}_{3 / 2}^{\mathrm{o}}$ & 96908 & 97091 & $913(50)$ \\
\hline \multirow[t]{2}{*}{12} & $2 \mathrm{Mg} \mathrm{I}$ & $3 s^{2}$ & ${ }^{1} \mathrm{~S}_{0}$ & $3 s 3 p$ & ${ }^{1} \mathrm{P}_{1}^{o}$ & 35051 & 35050 & $85(1)$ \\
\hline & & & & $3 s 4 p$ & ${ }^{1} \mathrm{P}_{1}^{o}$ & 49347 & 49277 & $80(1)$ \\
\hline 13 & $3 \mathrm{Al} \mathrm{II}$ & $3 s^{2}$ & ${ }^{1} \mathrm{~S}_{0}$ & $3 s 3 p$ & ${ }^{1} \mathrm{P}_{1}^{o}$ & 59852 & 59800 & $270(1)$ \\
\hline \multirow[t]{2}{*}{14} & $4 \mathrm{Si} \mathrm{II}$ & $3 s^{2} 3 p$ & ${ }^{2} \mathrm{P}_{1 / 2}^{o}$ & $3 s 3 p^{2}$ & ${ }^{2} \mathrm{D}_{3 / 2}$ & 55309 & 54655 & $520(30)$ \\
\hline & & & & $3 s^{2} 4 s$ & ${ }^{2} \mathrm{~S}_{1 / 2}$ & 65500 & 54675 & $50(30)$ \\
\hline \multirow[t]{7}{*}{22} & $2 \mathrm{Ti}$ II & $3 d^{2} 4 s$ & ${ }^{4} \mathrm{~F}_{3 / 2}$ & $3 d^{2} 4 p$ & ${ }^{4} \mathrm{G}_{5 / 2}^{o}$ & 29544 & 28097 & $396(50)$ \\
\hline & & & & $3 d^{2} 4 p$ & ${ }^{4} \mathrm{~F}_{3 / 2}^{o}$ & 30837 & 29401 & $541(50)$ \\
\hline & & & & $3 d^{2} 4 p$ & ${ }^{4} \mathrm{~F}_{5 / 2}^{o}$ & 30959 & 29521 & $673(50)$ \\
\hline & & & & $3 d^{2} 4 p$ & ${ }^{4} \mathrm{D}_{1 / 2}^{o}$ & 32532 & 31143 & $677(50)$ \\
\hline & & & & $3 d^{2} 4 p$ & ${ }^{4} \mathrm{D}_{3 / 2}^{o}$ & 32603 & 31227 & $791(50)$ \\
\hline & & & & $3 d 4 s 4 p$ & ${ }^{4} \mathrm{D}_{1 / 2}^{o}$ & 52339 & 50889 & $-1564(150)$ \\
\hline & & & & $3 d 4 s 4 p$ & ${ }^{4} \mathrm{~F}_{3 / 2}^{o}$ & 52330 & 51341 & $-1783(150)$ \\
\hline 22 & $2 \mathrm{Ti}$ III & $3 d^{2}$ & ${ }^{3} \mathrm{~F}_{2}$ & $3 d 4 p$ & ${ }^{3} \mathrm{D}_{1}^{o}$ & 77000 & 80558 & $-1644(150)$ \\
\hline \multirow[t]{3}{*}{24} & $4 \mathrm{Cr}$ II & $3 d^{5}$ & ${ }^{6} \mathrm{~S}_{5 / 2}$ & $3 d^{4} 4 p$ & ${ }^{6} \mathrm{P}_{3 / 2}^{o}$ & 48398 & 48684 & $-1360(150)$ \\
\hline & & & & $3 d^{4} 4 p$ & ${ }^{6} \mathrm{P}_{5 / 2}^{o}$ & 48491 & 48790 & $-1280(150)$ \\
\hline & & & & $3 d^{4} 4 p$ & ${ }^{6} \mathrm{P}_{7 / 2}^{o}$ & 48632 & 48947 & $-1110(150)$ \\
\hline \multirow[t]{6}{*}{25} & $5 \mathrm{Mn}$ II & $3 d^{5} 4 s$ & ${ }^{7} \mathrm{~S}_{3}$ & $3 d^{5} 4 p$ & ${ }^{7} \mathrm{P}_{2}^{o}$ & 38366 & 38424 & $869(150)$ \\
\hline & & & & $3 d^{5} 4 p$ & ${ }^{7} \mathrm{P}_{3}^{o}$ & 38543 & 38585 & $1030(150)$ \\
\hline & & & & $3 d^{5} 4 p$ & ${ }^{7} \mathrm{P}_{4}^{o}$ & 38807 & 38814 & $1276(150)$ \\
\hline & & & & $3 d^{4} 4 s 4 p$ & ${ }^{7} \mathrm{P}_{2}^{o}$ & 83256 & 83363 & $-3033(450)$ \\
\hline & & & & $3 d^{4} 4 s 4 p$ & ${ }^{7} \mathrm{P}_{3}^{o}$ & 83376 & 83559 & $-2825(450)$ \\
\hline & & & & $3 d^{4} 4 s 4 p$ & ${ }^{7} \mathrm{P}_{4}^{o}$ & 83529 & 83818 & $-2556(450)$ \\
\hline \multirow[t]{2}{*}{28} & $8 \mathrm{Ni}$ II & $3 d^{9}$ & ${ }^{2} \mathrm{D}_{5 / 2}$ & $3 d^{8} 4 p$ & ${ }^{2} \mathrm{~F}_{7 / 2}^{o}$ & 57080 & 56067 & $-700(250)$ \\
\hline & & & & $3 d^{8} 4 p$ & ${ }^{2} \mathrm{D}_{5 / 2}^{o}$ & 57420 & 56520 & $-1400(250)$ \\
\hline 32 & 2 Ge II & $4 s^{2} 4 p$ & ${ }^{3} \mathrm{P}_{1 / 2}^{o}$ & $4 s^{2} 5 s$ & ${ }^{2} \mathrm{~S}_{1 / 2}$ & 62402 & 62870 & $-630(40)$ \\
\hline
\end{tabular}

transitions between the states of the same fine structure multiplet [37]. It was argued that the anomalies in the fine structure intervals lead to enhancement of the sensitivity of the transition frequencies to variation of the fine structure constant. This idea is similar to what what suggested before for laboratory experiments [38].

It is also important to include molecular lines into analysis. This would allow to study variation of the ratio of the electron to proton mass $(\mu)$. Most of grand unification models suggest that the variation of mass ratio and $\alpha$ are related and mass ratio is changing faster than $\alpha$. Therefore it might be easier to find manifestations of variation of mass ratio. There are already published results of such analysis performed by different groups [39, 40] but as for the case of $\alpha$ the results are controversial but the other way around. Those who claim variation of $\alpha$ see 
no variation of $\mu$ and vise versa. Here again more study is needed and there are some new interesting suggestions (see, e.g. 41]).

\section{CONCLUSION}

The many-multiplet method which is based on the analysis of the frequencies of strong electric dipole transitions in atoms and ions found in gas clouds intersecting the sight line from Earth to distant quasars prove to be a useful tool for the search of the variation of the fine structure constant in quasar absorption spectra. The method relies on atomic calculations to reveal the dependence of atomic frequencies on the fine structure constant. Critical compilation of the all relevant calculations performed by our and some other groups and presented in this paper can serve as a reference point for future analysis. The results of the analysis so far is controversial. The analysis of the data from Keck telescope in Hawaii indicate that $\alpha$ might be smaller in early Universe while similar analysis of the data from the VLT telescope in Chile performed by different groups of researchers gives null result. Both analysis use the same MM method and the same atomic calculations. This means the the reason for disagreement is probably not relevant to atomic calculations and is rather in the data or the analysis. Recent re-analysis of the VLT data performed by the authors of the analysis of the Keck data pointed to some serious problems in the method used by the other group. Further revision of the VLT data is needed to resolve all problems in the analysis and disagreement in the results.

\section{Acknowledgment}

The work was supported in part by the Australia Research Council.
[1] J-P. Uzan, Rev. Mod. Phys. 75, 403 (2003).

[2] V. V. Flambaum, Int. J. Mod. Phys. A 22, 4937 (2007).

[3] J. K. Webb, V. V. Flambaum, C. W. Churchill, M. J. Drinkwater, and J. D. Barrow, Phys. Rev. Lett. 82, 884 (1999).

[4] J. K. Webb, M. T. Murphy, V. V. Flambaum, V. A. Dzuba, J. D. Barrow, C. W. Churchill, J. X. Prochaska, and A. M. Wolfe, Phys. Rev. Lett. 87, 091301 (2001).

[5] M. T. Murphy, J. K. Webb, V. V. Flambaum, V. A. Dzuba, C. W. Churchill, J. X. Prochaska, J. D. Barrow, and A. M. Wolfe, Not. R. Astron. Soc. 327, 1208 (2001).

[6] M. T. Murphy, J. K. Webb, V. V. Flambaum, C. W. Churchill, and J. X. Prochaska, Mon. Not. R. Astron. Soc. 327, 1223 (2001).

[7] M. T. Murphy, J. K. Webb, V. V. Flambaum, C. W. Churchill, and J. X. Prochaska, Mon. Not. R. Astron. Soc. 327, 1237 (2001).

[8] M. T. Murphy, J. K. Webb, V. V. Flambaum, M. J. Drinkwater, F. Combes, and T. Wiklind, Mon. Not. R. Astron. Soc. 327, 1244 (2001).

[9] J. K. Webb, M. T. Murphy, V. V. Flambaum, and S. G. Curran, ApSS, 283, 565 (2003).

[10] M. T. Murphy, J. K. Webb, V. V. Flambaum, Mon. Not. R. Astron. Soc. 345, 609 (2003).

[11] J. K. Webb, V. V. Flambaum, and S.G. Curran, Astrophysics and space science (ApSS) 283, 577 (2003).

[12] R. Srianand, H. Chand, P. Petitjean, and B. Aracil, Phys. Rev. Lett. 92, 121302 (2004).

[13] S. A. Levshakov et al., Astron. Astrophys, 434, 827 (2005).

[14] S. A. Levshakov et al., Astron. Astrophys, 449, 879 (2006).

[15] M. T. Murphy, J. K. Webb, V. V. Flambaum, Phys. Rev. Lett. 99, 239001 (2007).

[16] M. T. Murphy , J. K. Webb , V. V. Flambaum, astro-ph/0612407, accepted to MNRAS.

[17] V. A. Dzuba, V. V. Flambaum, and J. Webb, Phys. Rev. Lett., 82, 888 (1999).
[18] V. A. Dzuba, V. V. Flambaum, J. Webb, Phys. Rev. A, 59, 230 (1999).

[19] V. A. Dzuba, V. V. Flambaum, M. T. Murphy and J. K. Webb, Phys. Rev. A, 63, 042509 (2001).

[20] V. A. Dzuba, V. V. Flambaum, M. G. Kozlov, and M. Marchenko, Phys. Rev. A, 66, 022501 (2002).

[21] J. C. Berengut, V. A. Dzuba, V. V. Flambaum, and M. V. Marchenko, Phys. Rev. A, 70, 064101 (2004).

[22] J. C. Berengut, V. V. Flambaum, and M. G. Kozlov Phys. Rev. A, 72, 044501 (2005).

[23] V. A. Dzuba, and V. V. Flambaum, Phys. Rev. A, 71, 052509 (2005).

[24] J. C. Berengut, V. V. Flambaum, and M. G. Kozlov Phys. Rev. A, 73, 012504 (2006).

[25] V. A. Dzuba and W. R. Johnson, Phys. Rev. A, 76 062510 (2007).

[26] I. Savukov, V.A. Dzuba, Phys. Rev. A, 77, 042501 (2008).

[27] V. A. Dzuba and V. V. Flambaum, Phys. Rev. A, 77, 012514 (2008).

[28] V. A. Dzuba, V. V. Flambaum, P. G. Silvestrov, O. P. Sushkov, J. Phys. B: At. Mol. Phys., 20, 1399-1412 (1987).

[29] W. R. Johnson and J. Sapirstein, Phys. Rev. Lett. 57, 1126 (1986); W. R. Johnson, M. Idrees, and J. Sapirstein, Phys. Rev. A 35, 3218 (1987); W. R. Johnson, S. A. Blundell and J. Sapirstein, Phys. Rev. A 37, 307 (1988).

[30] V. A. Dzuba, V. V. Flambaum, and M. G. Kozlov, Phys. Rev. A, 54, 3948 (1996).

[31] V. A. Dzuba, Phys. Rev. A, 71, 032512 (2005).

[32] V. A. Dzuba and V. V. Flambaum, Phys. Rev. A, 75, 052504 (2007).

[33] S. G. Porsev, K. V. Koshelev, I. I. Tupitsyn, M. G. Kozlov, D. Reimers, and S. A. Levshakov, Phys. Rev. A, 76, 052507 (2007).

[34] P. Bogdanovich and G. Zukauskas, Sov. Phys. Collect. 23, 13 (1983).

[35] V. V. Flambaum, and J. S. M. Ginges, Phys. Rev. A, 72, 
052115 (2005).

[36] Ralchenko, Yu., Jou, F.-C., Kelleher, D.E., Kramida, A.E., Musgrove, A., Reader, J., Wiese, W.L., and Olsen, K. (2007). NIST Atomic Spectra Database (version 3.1.3), [Online]. Available: http://physics.nist.gov/asd3 [2007, September 18]. National Institute of Standards and Technology, Gaithersburg, MD.

[37] M. G. Kozlov, S. G. Porsev, S. A. Levshakov, D. Reimers, and P. Molaro, arXiv:0802.0269 (2008).
[38] V. A. Dzuba, and V. V. Flambaum, Phys. Rev. A, 72, 052514 (2005).

[39] E. Reinhold, R. Buning, U. Hollenstein, A. Ivanchik, P. Petitjean, and W. Ubachs, Phys. Rev. Lett. 96, 151101 (2006).

[40] V. V. Flambaum and M. G. Kozlov, Phys. Rev. Lett. 98, 240801 (2007)

[41] V. V. Flambaum, M. G. Kozlov, arXiv:0711.4536 (2007). 